\title{
Synthesis of 2,6-bis-polyfluoroalkyl-4H-pyran-4-ones
}

\author{
Danil S. Yachevskii,* Dmitry L. Chizhov, Kazimir I. Pashkevich, and Valery N. Charushin \\ I. Postovsky Institute of Organic Synthesis of RAS, \\ 22/20, S. Kovalevskaya / Akademicheskaya st., GSP-147, Ekaterinburg, 620219, Russia \\ E-mail:yad@ios.uran.ru
}

Dedicated to Prof. Oleg N. Chupakhin on his $70^{\text {th }}$ anniversary

(received 02 Nov 04; accepted 14 Jan 05; published on the web 22 Jan 05)

\begin{abstract}
A convenient synthesis is described of 2,6-bis-polyfluoroalkyl-4H-pyran-4-ones having symmetrical and asymmetrical structures, by dehydration of bis-polyfluoroalkyl- containing 1,3,5-triketones.
\end{abstract}

Keywords: 2,6-Bis-polyfluoroalkyl-4H-pyran-4-ones, bis-polyfluoroalkyl- containing 1,3,5triketones, dehydration, synthesis

\section{Introduction}

Polyfluoroalkyl- substituted heterocyclic compounds are of particular interest owing to their biological activity, specific properties and chemical reactivity. Such compounds may have significant applications in various fields (medicinal, ${ }^{1}$ agricultural, ${ }^{2}$ etc.). Hence, there is a need for the development of accessible and convenient methods for the introduction of polyfluoroalkyl groups into heterocyclic systems. One of the prospective directions for investigations is the synthesis of fluoroalkyl substituted 4H-pyran-4-ones ( $\gamma$-pyrones) which can be suitable precursors of polyfluoroalkylated heterocycles. In particular, recyclization of the title compounds with ammonia and primary amines can lead to $4-(1 \mathrm{H})$-pyridones or the corresponding pyridine derivatives, whose properties are widely covered in the literature. ${ }^{1 \mathrm{~b}, \mathrm{c}, 2 \mathrm{c}, 3}$ On the other hand, there are only limited data concerning polyfluoroalkyl containing $\gamma$-pyrones. ${ }^{4}$

In the present paper the cyclization of bis-polyfluoroalkyl-1,3,5-triketones $\mathbf{1 c}-\mathbf{e}, \mathbf{h}-\mathbf{j}$ (or their cyclic hydrates 1a,b,f,g) ${ }^{5}$ to form 2,6-bis-polyfluoroalkyl-4H-pyran-4-ones by the action of various dehydrating agents has been investigated, and the selection of the optimal conditions for the dehydration has been made. 


\section{Results and Discussion}

As reported earlier, the treatment of lithium salts of 1,3,5-triketones ("TK") with dilute mineral acids was accompanied by the formation of 2,6-bis-polyfluoroalkyl-4H-pyran-4-ones. ${ }^{5}$ However, under these conditions dehydration proceeded to the end only for di- and trifluoromethyl substituted TK, whereas with the higher substituents $\gamma$-pyrones were obtained as by-products. To accomplish the conversion of TK we have examined various dehydrating agent (among them $\mathrm{H}_{2} \mathrm{SO}_{4}$, polyphosphoric acid (PPA), methanolic $\mathrm{HCl}, \mathrm{P}_{4} \mathrm{O}_{10}$, trimethylsilyl phosphate $\left(\mathrm{Me}_{3} \mathrm{Si}\right)_{3} \mathrm{PO},{ }^{6}$ ethyl polyphosphate $\left.\left[\mathrm{EtOPO}_{2}\right]_{\mathrm{n}}{ }^{7}\right)($ Scheme 1).

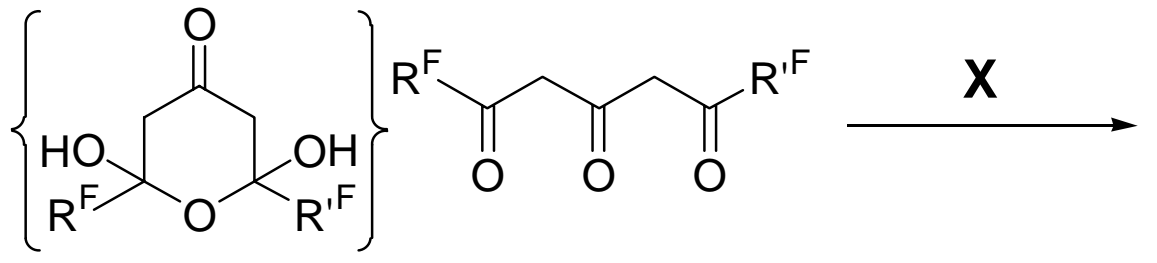

$1 a, b, f, g$ 1c-e,h-j<smiles>[R7]c1cc(=O)cc([R])o1</smiles>

$2 a-j$

$$
\mathbf{X}=\mathrm{H}_{2} \mathrm{SO}_{4}, \mathrm{PPA}, \mathrm{HCl} / \mathrm{MeOH},\left(\mathrm{Me}_{3} \mathrm{SiO}\right)_{3} \mathrm{PO},\left(\mathrm{EtOPO}_{2}\right)_{\mathrm{n}} / \mathrm{CHCl}_{3}
$$

\section{Scheme 1}

Employment of the concentrated sulfuric acid used earlier for the cyclization of nonfluorinated TK to give $\gamma$-pyrones, ${ }^{8}$ as well as PPA yielded unsatisfactory results - even after heating for several hours the starting TK was partially recovered (according to TLC data). Refluxing of TK with hydrochloric acid in methanol also appeared ineffective. The dehydration of TK in presence of $\mathrm{P}_{4} \mathrm{O}_{10}$ proceeded completely by heating over a period of $10-30 \mathrm{~min}$. in 50$75 \%$ yields, but was accompanied by the loss of a part of the $\gamma$-pyrones owing to adsorption on the dehydrating agent. For high-boiling compounds this problem was solved, in part, by vacuum distillation from the reaction mixture, whereas for the low-boiling ones it still led to big losses. Refluxing of TK and trimethylsilyl phosphate mixture in chloroform or without solvent led to compounds 2 in $60-70 \%$ yields. However, as ${ }^{1} \mathrm{H}$ - NMR data showed the $\gamma$-pyrones obtained were contaminated with silicon substances (mainly, hexamethyldisiloxane). Attempts to remove these impurities by distillation failed. Ethyl polyphosphate appeared to be the most effective dehydrating agent with regard to the isolation and yield of products formed. Thus, heating of TK in the presence of ethyl polyphosphate at reflux in chloroform during 20-60 minutes followed by workup of the reaction mixture with water resulted in the desired $\gamma$-pyrones in $75-95 \%$ yields (Table 1). 
The structures of the obtained compounds were confirmed by IR-, ${ }^{1} \mathrm{H}-$ and ${ }^{19} \mathrm{~F}$ - NMR spectroscopy, and elemental analysis. The IR spectra have bands in the region $1680-1620 \mathrm{~cm}^{-1}$ corresponding to vibrations of the $\mathrm{C}=\mathrm{C}-\mathrm{C}=\mathrm{O}$ conjugated system. Also an absorption maximum in the region of $3085-3060 \mathrm{~cm}^{-1}$ typical of $=\mathrm{C}-\mathrm{H}$ stretching vibrations is observed. The ${ }^{1} \mathrm{H}-$ NMR spectra of compounds 2a-e have sharp singlets at $\delta 6.66-6.87$ corresponding to the vinyl protons at $\mathrm{C}-3$ and $\mathrm{C}-5$. For the asymmetrically substituted $\gamma$-pyrones $2 \mathbf{f}-\mathbf{j}$ the signals of these protons are manifested as $\mathrm{AB}-$ systems with centers at $\delta$ 6.74-6.84 due to spin-spin coupling through the "W-conformation" 9 with the coupling constant ${ }^{4} \mathrm{~J} \mathrm{H}, \mathrm{H}=2.2-2.3 \mathrm{~Hz}$.

In conclusion, a convenient method for the preparation of 2,6-bis-polyfluoroalkyl-4H-pyran4-ones has been developed, based on the dehydration of bis-polyfluoroalkyl- containing 1,3,5triketones by using ethyl polyphosphate.

Table 1. Dehydration of TK by action of ethyl polyphosphate

\begin{tabular}{ccccc}
\hline Compd. & $\mathrm{R}^{\mathrm{F}}$ & $\mathrm{R}^{, \mathrm{F}}$ & $\begin{array}{c}\mathrm{bp}{ }^{\circ} \mathrm{C} \\
\left(\mathrm{mp}^{\circ} \mathrm{C}\right)\end{array}$ & $\begin{array}{c}\text { Yield, \% } \\
\mathrm{X}=\left(\mathrm{EtOPO}_{2}\right)_{\mathrm{n}}\end{array}$ \\
\hline 2a & $\mathrm{CHF}_{2}$ & $\mathrm{CHF}_{2}$ & $(58-59)$ & 81 \\
2b & $\mathrm{CF}_{3}$ & $\mathrm{CF}_{3}$ & $139-140$ & 73 \\
2c & $\mathrm{C}_{2} \mathrm{~F}_{4} \mathrm{H}$ & $\mathrm{C}_{2} \mathrm{~F}_{4} \mathrm{H}$ & 194 & 90 \\
2d & $\mathrm{C}_{4} \mathrm{~F}_{9}$ & $\mathrm{C}_{4} \mathrm{~F}_{9}$ & 183 & 89 \\
2e & $\mathrm{C}_{6} \mathrm{~F}_{13}$ & $\mathrm{C}_{6} \mathrm{~F}_{13}$ & $237(28)$ & 87 \\
$\mathbf{2 f}$ & $\mathrm{CHF}_{2}$ & $\mathrm{CF}_{3}$ & 177 & 95 \\
2g & $\mathrm{C}_{2} \mathrm{~F}_{4} \mathrm{H}$ & $\mathrm{CF}_{3}$ & 180 & 84 \\
2h & $\mathrm{C}_{3} \mathrm{~F}_{7}$ & $\mathrm{CF}_{3}$ & 161 & 78 \\
2i & $\mathrm{C}_{4} \mathrm{~F}_{9}$ & $\mathrm{CF}_{3}$ & 150 & 79 \\
2j & $\mathrm{C}_{6} \mathrm{~F}_{13}$ & $\mathrm{CF}_{3}$ & 178 & 93 \\
\hline
\end{tabular}

\section{Experimental Section}

General Procedures. Melting points are uncorrected. NMR spectra $(\delta, \mathrm{ppm} ; J, \mathrm{~Hz})$ were recorded on Bruker DRX $400\left(400.13 \mathrm{MHz},{ }^{1} \mathrm{H}\right)$ and Tesla BS-587A $\left(75.3 \mathrm{MHz},{ }^{19} \mathrm{~F}\right)$ instruments in $\mathrm{CDCl}_{3}$ solutions with $\mathrm{Me}_{4} \mathrm{Si}(1 \mathrm{H})$ and $\mathrm{C}_{6} \mathrm{~F}_{6}\left({ }^{19} \mathrm{~F}\right)$ as internal standards. ${ }^{19} \mathrm{~F}-\mathrm{NMR}$ 
data are given only for $\mathrm{CF}_{2} \mathrm{H}, \mathrm{CF}_{3}$ and $\mathrm{C}_{2} \mathrm{~F}_{4} \mathrm{H}$ substituents. Infrared spectra were recorded in the $4000-400 \mathrm{~cm}^{-1}$ range on a Specord 75IR spectrometer. Elemental analyses $(\mathrm{C}, \mathrm{H}, \mathrm{F})$ were measured using a Perkin Elmer Elemental Analyzer 2400.

Ethyl polyphosphate $\left[\mathrm{EtOPO}_{2}\right]_{\mathrm{n}}(\mathrm{Mr} \approx 432)$ was obtained by a literature method. ${ }^{8}$ 2,6-Bispolyfluoroalkyl-4H-pyran-4-ones were prepared according to the procedures given below. The course of the reaction was monitored by TLC on Silufol UV-254 plates using chloroform as eluent.

\section{General procedure for the synthesis of compounds $\mathbf{2} a-\mathbf{j}$}

The mixture of 1,3,5-triketone (10 mmol), ethyl polyphosphate $(7 \mathrm{mmol})$ and chloroform $(10 \mathrm{ml})$ was heated under reflux over a period of 20-60 minutes, samples being withdrawn at intervals. The samples were treated with a solution of copper acetate and extracted with $\mathrm{AcOEt}_{\text {or }} \mathrm{Et}_{2} \mathrm{O}$. The extract was analyzed by TLC. Completion of the reaction was determined by the disappearance of a green spot of the copper triketonate $\left(\mathrm{R}_{\mathrm{f}}=0-0.1\right)$. Then the reaction mixture was quenched with water, extracted with chloroform $(3 \times 10 \mathrm{ml})$ and dried over $\mathrm{Na}_{2} \mathrm{SO}_{4}$. Solvent was distilled off using a fractionating column to prevent loss of $\delta$-pyrones. The residue was distilled as usual, with the exception of compounds $\mathbf{2 a}, \mathbf{e}$ which were recrystallized from $\mathrm{CHCl}_{3}$ and carbogal, respectively without distillation.

2,6-Bis-(difluoromethyl)-4H-pyran-4-one (2a). Pale yellow crystals from chloroform; IR (Nujol, $\left.\mathrm{cm}^{-1}\right)$ 3085, 1680, 1640; 1H-NMR (CDCl3) $\delta 6.66(\mathrm{~s}, 2 \mathrm{H}), 6.42(\mathrm{t}, \mathrm{J}=53.2 \mathrm{~Hz}, 2 \mathrm{H}) ;{ }^{19} \mathrm{~F}-$ NMR $\left(\mathrm{CDCl}_{3}\right) \delta 38.18(\mathrm{~d}, \mathrm{~J}=53.5 \mathrm{~Hz}, 4 \mathrm{~F})$; Anal. Calcd. for $\mathrm{C}_{7} \mathrm{H}_{4} \mathrm{~F}_{4} \mathrm{O}_{2}: \mathrm{C}, 42.87 ; \mathrm{H}, 2.06 ; \mathrm{F}$, 38.75. Found: C, 42.56; H, 2.20; F, 39.04\%.

2,6-Bis-(trifluoromethyl)-4H-pyran-4-one (2b). Pale yellow liquid; IR (film, $\mathrm{cm}^{-1}$ ) 3065, 1680, $1650 ;{ }^{1} \mathrm{H}-\mathrm{NMR}\left(\mathrm{CDCl}_{3}\right) \delta 6.81(\mathrm{~s}, 2 \mathrm{H}) ;{ }^{19} \mathrm{~F}-\mathrm{NMR}\left(\mathrm{CDCl}_{3}\right) \delta 90.33(\mathrm{~s}, 6 \mathrm{~F})$; Anal. Calcd. for $\mathrm{C}_{7} \mathrm{H}_{2} \mathrm{~F}_{6} \mathrm{O}_{2}$ : C, 36.23; H, 0.87; F, 49.12. Found: C, 36.60; H, 0.97; F, 48.98\%.

2,6-Bis-(1,1,2,2-tetrafluoroethyl)-4H-pyran-4-one (2c). Pale yellow liquid; IR (film, $\mathrm{cm}^{-1}$ ) 3070, 1680, 1645; ${ }^{1} \mathrm{H}-\mathrm{NMR}\left(\mathrm{CDCl}_{3}\right) \delta 6.81(\mathrm{~s}, 2 \mathrm{H}), 6.06\left(\mathrm{tt}, 2 \mathrm{~J}=52.9 \mathrm{~Hz},{ }^{3} \mathrm{~J}=2.8 \mathrm{~Hz}, 2 \mathrm{H}\right) ;{ }^{19} \mathrm{~F}-$ NMR $\left(\mathrm{CDCl}_{3}\right) \delta 26.58\left(\mathrm{dt}, 2 \mathrm{~J}=52.7 \mathrm{~Hz},{ }^{3} \mathrm{~J}=3.9 \mathrm{~Hz}, 4 \mathrm{~F}, \mathrm{CF}_{2} \mathrm{CF}_{2} \mathrm{H}\right), 40.79-40.94(\mathrm{~m}, 4 \mathrm{~F}$, $\left.\mathrm{CF}_{2} \mathrm{CF}_{2} \mathrm{H}\right)$; Anal. Calcd. for $\mathrm{C}_{9} \mathrm{H}_{4} \mathrm{~F}_{8} \mathrm{O}_{2}$ : C, 36.51; H, 1.36; F, 51.33. Found: $\mathrm{C}, 36.22 ; \mathrm{H}, 1.23$; F, $51.93 \%$.

2,6-Bis-(1,1,2,2,3,3,4,4,4-nonafluorobutyl)-4H-pyran-4-one (2d). Yellow liquid; IR (film, $\mathrm{cm}^{-1}$ ) 3060, 1680, 1650, 1625; ${ }^{1} \mathrm{H}-\mathrm{NMR}\left(\mathrm{CDCl}_{3}\right) \delta 6.87(\mathrm{~s}, 2 \mathrm{H})$; Anal. Calcd. for $\mathrm{C}_{13} \mathrm{H}_{2} \mathrm{~F}_{18} \mathrm{O}_{2}$ : C, 29.34; H, 0.38; F, 64.26. Found: C, 29.33; H, 0.56; F, 64.35\%.

2,6-Bis-(1,1,2,2,3,3,4,4,5,5,6,6,6-tridecafluorohexyl)-4H-pyran-4-one (2e). Cream-colored flake-like crystals; IR (film, $\left.\mathrm{cm}^{-1}\right) 3080,1690,1655 ;{ }^{1} \mathrm{H}-\mathrm{NMR}\left(\mathrm{CDCl}_{3}\right) \delta 6.86$ (s, 2H); Anal. Calcd. for $\mathrm{C}_{17} \mathrm{H}_{2} \mathrm{~F}_{26} \mathrm{O}_{2}$ : C, 27.89; H, 0.28; F, 67.47. Found: C, 27.99; H, 0.15; F, 67.12\%.

2-(Difluoromethyl)-6-(trifluoromethyl)-4H-pyran-4-one (2f). Pale yellow liquid; IR (film, $\mathrm{cm}^{-1}$ ) 3070, 1680, 1640; ${ }^{1} \mathrm{H}-\mathrm{NMR}\left(\mathrm{CDCl}_{3}\right) \delta 6.74 \pm 0.01(\mathrm{AB}, \mathrm{J}=2.2 \mathrm{~Hz}, 2 \mathrm{H}), 6.43(\mathrm{t}, \mathrm{J}=53.3 \mathrm{~Hz}$, 
$2 \mathrm{H}) ;{ }^{19} \mathrm{~F}-\mathrm{NMR}\left(\mathrm{CDCl}_{3}\right) \delta 90.39(\mathrm{~s}, 3 \mathrm{~F}), \delta 37.92(\mathrm{~d}, \mathrm{~J}=53.7 \mathrm{~Hz}, 2 \mathrm{~F})$; Anal. Calcd. for $\mathrm{C}_{7} \mathrm{H}_{3} \mathrm{~F}_{5} \mathrm{O}_{2}$ : C, 39.27; H, 1.41; F, 44.37. Found: C, 39.40; H, 1.35; F, 44.32\%.

2-(1,1,2,2-Tetrafluoroethyl)-6-(trifluoromethyl)-4H-pyran-4-one (2g). Yellow liquid; IR (film, $\left.\mathrm{cm}^{-1}\right) 3065,1680,1645 ;{ }^{1} \mathrm{H}-\mathrm{NMR}\left(\mathrm{CDCl}_{3}\right) \delta 6.80 \pm 0.01(\mathrm{AB}, \mathrm{J}=2.2 \mathrm{~Hz}, 2 \mathrm{H}), 6.0(\mathrm{tt}, 2 \mathrm{~J}=$ $\left.53.0 \mathrm{~Hz},{ }^{3} \mathrm{~J}=2.8 \mathrm{~Hz}, 2 \mathrm{H}\right) ;{ }^{19} \mathrm{~F}-\mathrm{NMR}\left(\mathrm{CDCl}_{3}\right) \delta 90.16(\mathrm{~s}, 3 \mathrm{~F}) ; 26.62\left(\mathrm{dt},{ }^{2} \mathrm{~J}=53.7 \mathrm{~Hz},{ }^{3} \mathrm{~J}=3.9 \mathrm{~Hz}\right.$, $\left.2 \mathrm{~F}, \mathrm{CF}_{2} \mathrm{CF}_{2} \mathrm{H}\right), 40.92-41.07\left(\mathrm{~m}, 2 \mathrm{~F}, \mathrm{CF}_{2} \mathrm{CF}_{2} \mathrm{H}\right)$; Anal. Calcd. for $\mathrm{C}_{8} \mathrm{H}_{3} \mathrm{~F}_{7} \mathrm{O}_{2}: \mathrm{C}, 36.38 ; \mathrm{H}, 1.14 ; \mathrm{F}$, 50.36. Found: C, 36.50; H, 1.22; F, 50.49\%.

2-(1,1,2,2,3,3,3-Heptafluoropropyl)-6-(trifluoromethyl)-4H-pyran-4-one (2h). Pale yellow liquid; IR (film, $\mathrm{cm}^{-1}$ ) 3065, 1685, 1650, 1625; ${ }^{1} \mathrm{H}-\mathrm{NMR}\left(\mathrm{CDCl}_{3}\right) \delta 6.84 \pm 0.01(\mathrm{AB}, \mathrm{J}=2.2 \mathrm{~Hz}$, $2 \mathrm{H}) ;{ }^{19} \mathrm{~F}-\mathrm{NMR}(\mathrm{CDCl} 3) \delta 90.12$ (s, 3F); Anal. Calcd. for $\mathrm{C}_{9} \mathrm{H}_{2} \mathrm{~F}_{10} \mathrm{O}_{2}: \mathrm{C}, 32.55 ; \mathrm{H}, 0.61 ; \mathrm{F}, 57.21$. Found: C, 32.61; H, 0.70; F, 56.89\%.

2-(1,1,2,2,3,3,4,4,4-Nonafluorobutyl)-6-(trifluoromethyl)-4H-pyran-4-one (2i). Yellow liquid; IR (film, $\left.\mathrm{cm}^{-1}\right) 3060,1680,1650,1625 ;{ }^{1} \mathrm{H}-\mathrm{NMR}\left(\mathrm{CDCl}_{3}\right) \delta 6.84 \pm 0.01(\mathrm{AB}, \mathrm{J}=2.2 \mathrm{~Hz}, 2 \mathrm{H}) ;{ }^{19} \mathrm{~F}-$ NMR (CDCl3) $\delta 90.20$ (s, 3F); Anal. Calcd. for $\mathrm{C}_{10} \mathrm{H}_{2} \mathrm{~F}_{12} \mathrm{O}_{2}: \mathrm{C}, 31.43 ; \mathrm{H}, 0.53 ; \mathrm{F}, 59.66$. Found: C, 31.68; H, 0.44; F, 60.01\%.

2-(1,1,2,2,3,3,4,4,5,5,6,6,6-Tridecafluorohexyl)-6-(trifluoromethyl)-4H-pyran-4-one (2j). Yellow liquid; IR (film, $\mathrm{cm}^{-1}$ ) 3060, 1680, 1650, 1620; ${ }^{1} \mathrm{H}-\mathrm{NMR}\left(\mathrm{CDCl}_{3}\right) \delta 6.84 \pm 0.01(\mathrm{AB}, \mathrm{J}=2.2 \mathrm{~Hz}$, $2 \mathrm{H}) ;{ }^{19} \mathrm{~F}-\mathrm{NMR}\left(\mathrm{CDCl}_{3}\right) \delta 90.12(\mathrm{~s}, 3 \mathrm{~F})$; Anal. Calcd. for $\mathrm{C}_{12} \mathrm{H}_{2} \mathrm{~F}_{16} \mathrm{O}_{2}$ : C, 29.90; H, 0.42; F, 63.05. Found: C, 29.47; H, 0.39; F, 62.79\%.

\section{References}

1. (a) Welch, J.T. Tetrahedron 1987, 43, 3127. (b) Adam, S. ibid. 1989, 45, 1409. (c) McInally, T.; Tinker, A. C. J. Chem. Soc., Perkin Trans. 1 1988, 1837.

2. (a) Lee, L.F.; Sing, Y.L.L.; Wong, S.C. PCT Int. Appl. WO 93 11,112, 1993; Chem. Abstr. 1993, 119, 249850d. (b) Lee, L.F.; Stikes, G.L.; Molyneaux, J.M.; Sing, Y.L.; Chupp, J.P.; Woodard, S.S. J. Org. Chem. 1990, 55, 2872. (c) Lee, L.F.; Normansell, J.E. ibid. 1990, 55, 2964. (d) Bruner, H.G. Eur. Pat. Appl. EP 353,187 1990; Chem. Abstr. 1990, 113, 40462k.

3. (a) Lee, L.F.; Sing, Y.L. J. Org. Chem. 1990, 55, 380. (b) Vasil'ev, L.S., Azarevich, O.G.; Bogdanov, V.S., Ugrak, B.I.; Bochkareva, M.N., Dorokhov, V.A. Zh. Org. Khim. 1994, 30, 1702.

4. (a) England, D.C. J. Org. Chem. 1981, 46, 147. (b) Yagihara, H.; Goto, Y., Masamoto, K.; Morishima, Y.; Osabe, H. Ger. Offen, DE 3,707,828 1987; Chem. Abstr. 1988, 108, 131581m. (c) Lee, L.F.; Miller, M.L. Eur. Pat. Appl. EP 182,769 1986; Chem. Abstr. 1956, 105, 190960v. (d) Lee, L.F.; Spear, K.L.; Ruminski, P.G.; Dhingra, O.P. Eur. Pat. Appl. EP 276,204 1988; Chem. Abstr. 1989, 110, 57519v. (e) Tyvorskii, I.T.; Bobrov D.N.; Kulinkovich, O.G. Tetrahedron 1998, 54, 2819. 
5. Yachevskii, D.S.; Chizhov, D.L.; Ratner, V.G.; Pashkevich, K.I. Russ. Chem. Bull. 2001, 50, 1233.

6. Feher, F.; Kuhlborsch, G.; Blumke, A.; Keller, H.; Zippert, K. Ber. 1957, 90, 134.

7. Pollmann, W.; Schramm, G. Biochem. Biophys. Acta 1964, 1, 80.

8. (a) Miles, M.L.; Harris, T.M.; Hauser, C.R. J. Org. Chem. 1965, 30, 1007. (b) Marcus, E.; Chan, K.I., Strow, C.B. ibid. 1966, 31, 1369. (c) Wittek, P.J.; Hindley, K.B.; Harris, T.M. ibid. 1973, 38, 896.

9. Jackman, L.M.; Sternhell, S. Applications of NMR Spectroscopy in Organic Chemistry, $2^{\text {nd }}$ Edn; Pergamon Press: New York, 1969. 